(C) 2020 Wiley-VCH GmbH

\title{
ADVANCED MATERIALS INTERFACES
}

Supporting Information

for Adv. Mater. Interfaces, DOI: 10.1002/admi.202001091

Ultrahigh Infrared Photoresponse in Titanium Sesquioxide at Mott-Insulator Transition

Sukanta Nandi, Rahul Tripathi, Gobinda Das Adhikary, Pramod Kumar, and Abha Misra* 


\section{Supporting information}

\section{Ultrahigh Infrared Photoresponse in Titanium Sesquioxide at Mott-insulator transition}

Sukanta Nandi ${ }^{\mathrm{a}}$, Rahul Tripathi ${ }^{\mathrm{a}}$, Gobinda Das Adhikary ${ }^{\mathrm{b}}$, Pramod Kumar $^{\mathrm{c}}$ and Abha Misra ${ }^{\mathrm{a} *}$

${ }^{a}$ Department of Instrumentation and Applied Physics, Indian Institute of Science, Bangalore, Karnataka, India - 560012.

${ }^{\mathrm{b}}$ Department of Materials Engineering, Indian Institute of Science, Bangalore, Karnataka, India 560012.

${ }^{\mathrm{C}}$ Department of Chemistry, Sri Aurobindo College, (Delhi University), Shivalik, Malviya Nagar, New Delhi-110017.

*Email: abha.misra1@gmail.com

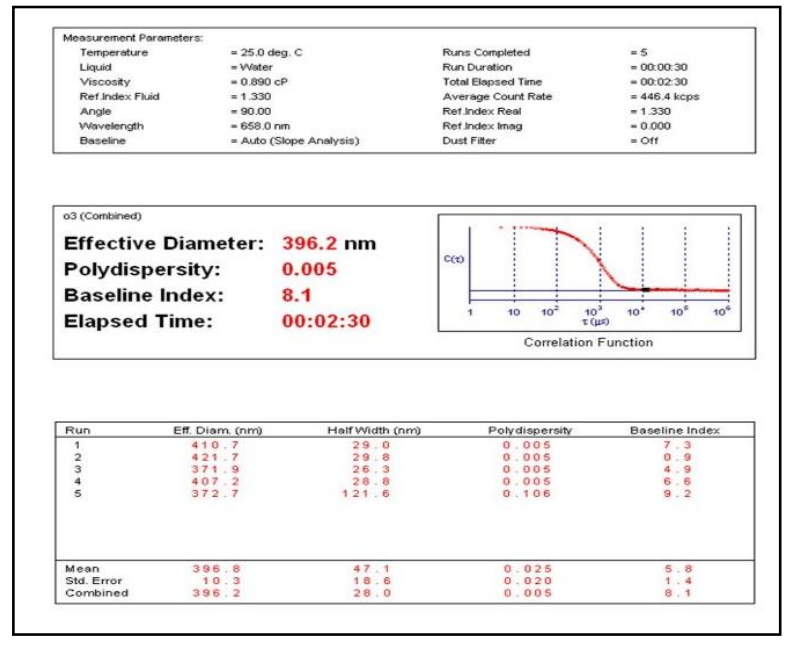

Figure S1. Zeta potential analysis (Zeta Phase Analysis Light Scattering) of TNPs. 


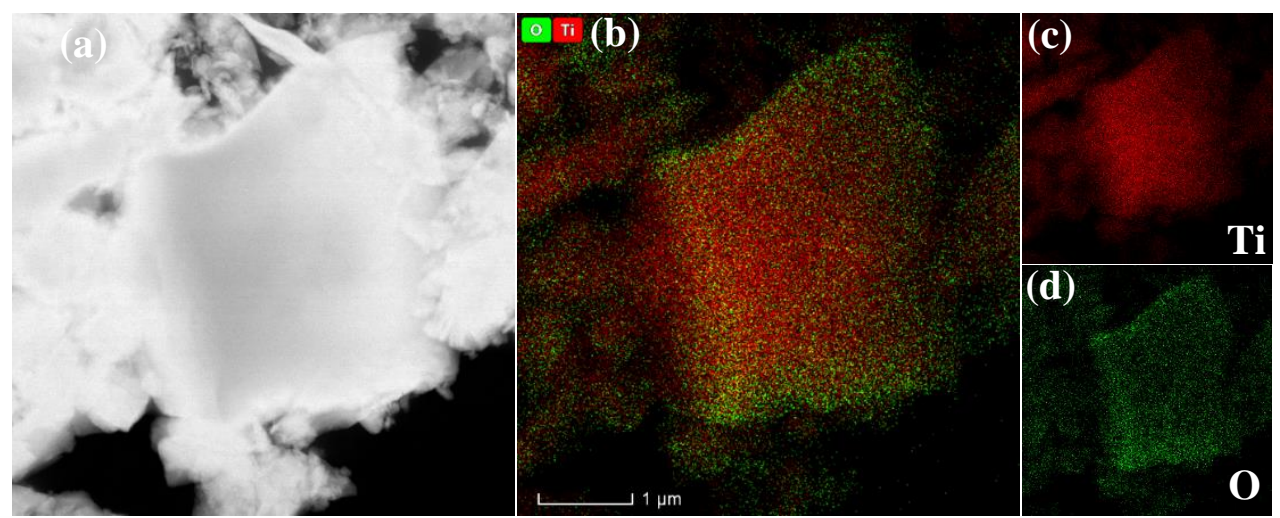

Figure S2. (a) Scanning TEM image and (b,c and d) the corresponding elemental mapping of a TNP.

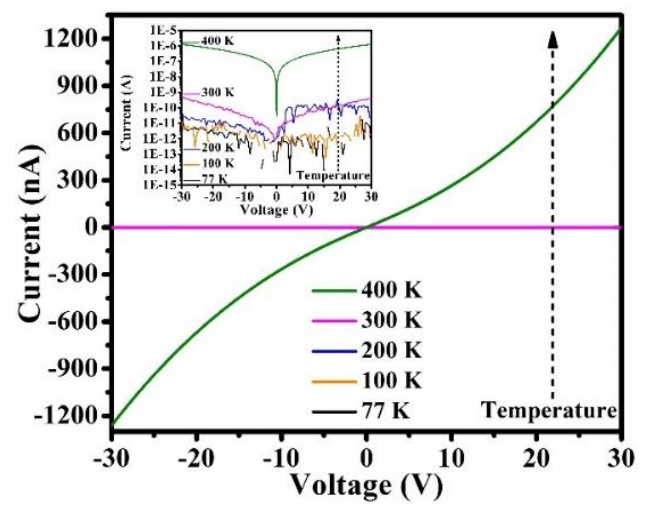

Figure S3. (a) $I-V$ with temperature sweep from $77-400 \mathrm{~K}$ for $50 \mu \mathrm{m}$ channel length device (inset: $I-V$ in $\log -$ scale).

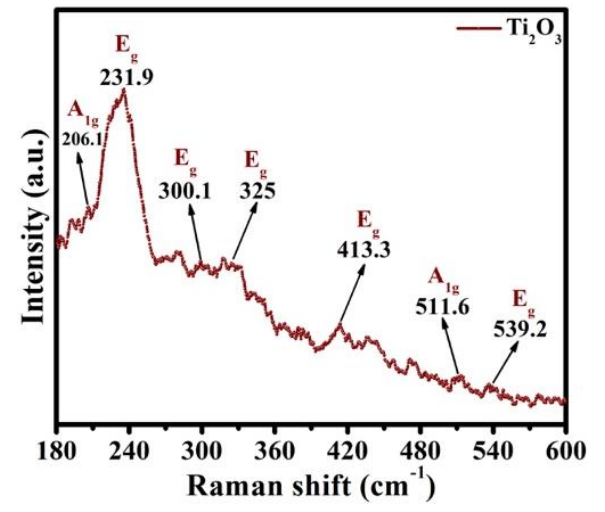

Figure S4. Raman spectra of TNPs at $300 \mathrm{~K}$. 


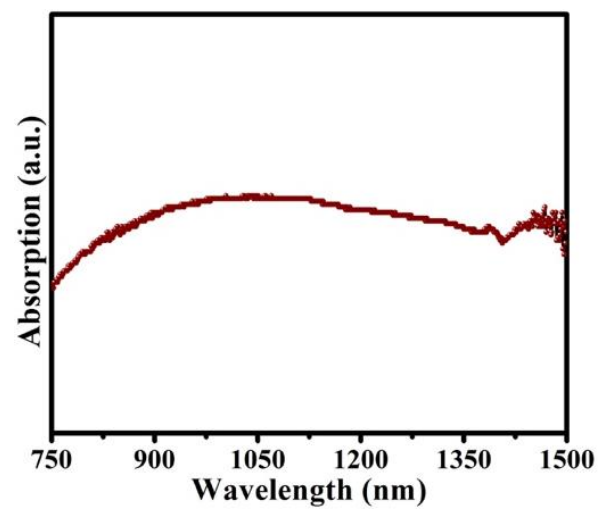

Figure S5. Absorption spectra of TNPs in the range 750-1500 nm.

(a)

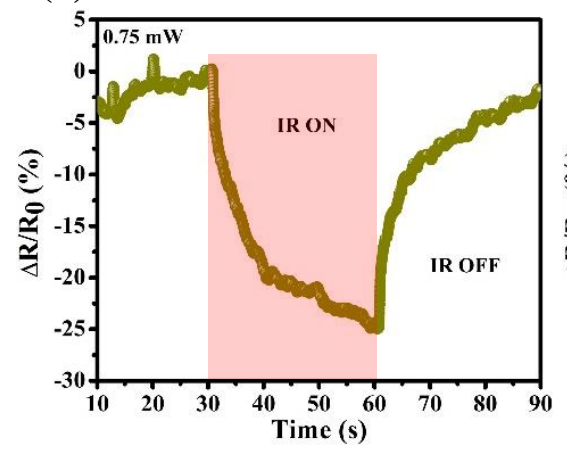

(b)

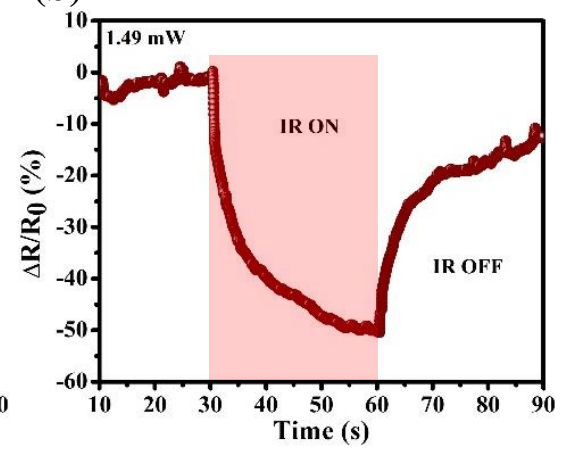

(c)

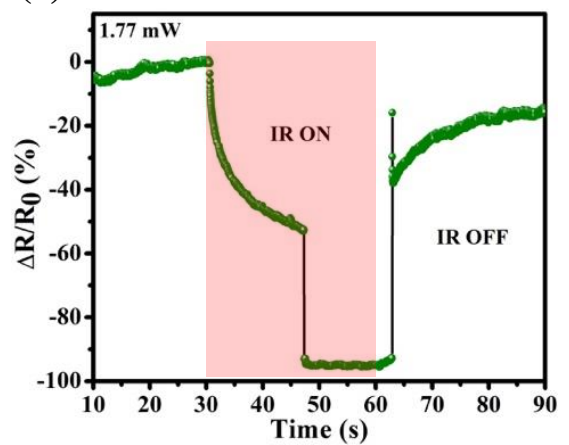

Figure S6. IR photoresponse at $400 \mathrm{~K}$ for different incident powers (a) 0.75 , (b) 1.49 and (c) $1.77 \mathrm{~mW}$.

Table 1

\begin{tabular}{|c|c|}
\hline Wavelength $(\mathrm{nm})$ & 1064 \\
\hline Output power from laser, $P_{\text {out }}(\mathrm{mW})$ & 265 \\
\hline Laser spot size, $A\left(\mathrm{~mm}^{2}\right)$ & $\sim 4.95$ \\
\hline Film area $\left(\mathrm{mm}^{2}\right)$ & $\sim 9.033$ \\
\hline Active area, $S\left(\mathrm{~mm}^{2}\right)$ & $\sim 0.033$ \\
\hline Incident power, $\left(P_{\text {inc }}=\frac{P_{\text {out }}}{A} \mathrm{x} S\right)(\mathrm{mW})$ & $\sim 1.77$ \\
\hline
\end{tabular}


Table 2

\begin{tabular}{|c|c|}
\hline Thermal condition $(\mathbf{K})$ & Value of lattice constant 'c' $(\AA)$ \\
\hline $300 \mathrm{~K}$ & 13.70369 \\
\hline $400 \mathrm{~K}$ & 13.72884 \\
\hline $300 \mathrm{~K} \mathrm{ramp}$ down & 13.68990 \\
\hline
\end{tabular}

